\title{
Young Women with Locally Advanced Breast Cancer Who Achieve Breast Conservation after Neoadjuvant Chemotherapy Have a Low Local Recurrence Rate
}

\author{
RAESHELL S. SWEETING, M.D. ${ }^{\star}$, NANCY KLAUBER-DEMORE, M.D. ${ }^{*} \S$, MICHAEL 0.

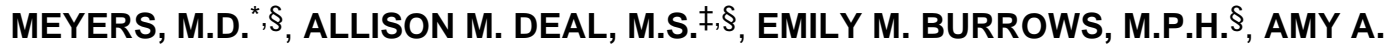 \\ DROBISH, M.A. $\S$, CAREY K. ANDERS, M.D. $\dagger, \S$, and LISA A. CAREY, M.D. ${ }^{\dagger} \S$ \\ "Division of Surgical Oncology, University of North Carolina School of Medicine at Chapel Hill, \\ Chapel Hill, North Carolina \\ tDivision of Medical Oncology, University of North Carolina School of Medicine at Chapel Hill, \\ Chapel Hill, North Carolina \\ FBiostatistics Core Facility, University of North Carolina School of Medicine at Chapel Hill, Chapel \\ Hill, North Carolina \\ $\S$ The Lineberger Comprehensive Cancer Center, University of North Carolina School of Medicine \\ at Chapel Hill, Chapel Hill, North Carolina
}

\begin{abstract}
Women with locally advanced breast cancer (LABC) who are breast conservation (BCT) candidates after neoadjuvant chemotherapy have the best long-term outcome and low localregional recurrence (LRR) rates. However, young women are thought to have a higher risk of LRR based on historical data. This study sought to evaluate LRR rates in young women who undergo BCT after neoadjuvant chemotherapy. We identified 122 women aged 45 years or younger with American Joint Committee on Cancer (AJCC) Stage II to III breast cancer, excluding T4d, treated with neoadjuvant chemotherapy from 1991 to 2007 from a prospective, Institutional Review Board-approved, single-institution database. Data were analyzed using Fisher eExact test, Wilcoxon tests, and the Kaplan-Meier method. Median follow-up was 6.4 years. Fifty-four (44\%) patients had BCT and 68 (56\%) mastectomy. Forty-six per cent were estrogen receptor-positivity and 28 per cent overexpressed Her2. Mean pretreatment T size was $5.6 \mathrm{~cm}$ in the BCT group and $6.7 \mathrm{~cm}$ in the mastectomy group $(P=0.04)$. LRR rates were no different after BCT compared with mastectomy (13 vs 18\%, $P=0.6)$. Higher posttreatment $\mathrm{N}$ stage $(P<0.001)$ and AJCC stage $(P=$ 0.008 ) were associated with LRR but not pretreatment staging. Disease-free survival was better for patients achieving BCT, with 5-year disease-free survival rates of 82 per cent (95\% CI, 69 to 90\%) compared with 58 per cent $(95 \% \mathrm{CI}, 45$ to $69 \%)$ for mastectomy $(P=0.03)$. Young women with LABC who undergo BCT after neoadjuvant chemotherapy appear to have similar LRR rates
\end{abstract}

\footnotetext{
Address correspondence and reprint requests to: Nancy Klauber-DeMore, M.D., Associate Professor of Surgery, 170 Manning Drive, CB \#7213, Chapel Hill, NC 27599. nancy_demore@ med.unc.edu.

Presented at the Annual Scientific Meeting and Postgraduate Course Program, Southeastern Surgical Congress, Chattanooga, TN, February 12-15, 2011.
} 
compared with those with mastectomy. This suggests that neoadjuvant chemotherapy may identify young women for whom BCT may have an acceptable risk of LRR.

Young women with breast cancer have been shown to be at higher risk of local recurrence when choosing breast conservation (BCT) as compared with mastectomy. A recent report demonstrated a 10-year local-regional recurrence (LRR) rate of 17.7 per cent with BCT compared with a 5.7 per cent rate with mastectomy plus radiation for young women with Stage II breast cancer who had not undergone neoadjuvant chemotherapy. ${ }^{1}$ Additionally, the Milan trial demonstrated a higher rate of recurrence in women aged younger than 45 years undergoing BCT versus mastectomy (1.05 per 100 women-years vs 0.18 per 100 womenyears). ${ }^{2}$ Other studies have shown that young women tend to have a poorer response to therapy, increased rates of LRR, and decreased overall survival (OS) when compared with older women. ${ }^{3}$ Higher rates of triple negative and Her2/neu-positive breast cancer in young women are thought to play a role in the more aggressive nature of breast cancer in young women. ${ }^{4,5}$

Neoadjuvant chemotherapy is now an accepted modality of treating locally advanced breast cancer. Prior studies have shown comparable rates of disease-free survival (DFS) and OS in patients undergoing neoadjuvant therapy as compared with adjuvant therapy. ${ }^{6,7}$

Additionally, neoadjuvant chemotherapy can be a means to convert patients with tumors too large for BCT to appropriate candidates for BCT if there is an adequate response. Many studies have demonstrated that there is no significant difference in LRR among patients who undergo BCT versus mastectomy after neoadjuvant therapy. ${ }^{8,}{ }^{8}$ We hypothesized that neoadjuvant chemotherapy would select out a group of young women whose tumors are responsive to chemotherapy and who may have more acceptable rates of local recurrence with BCT despite their young age based on more favorable tumor biology.

\section{Methods}

\section{Patients}

Patients were selected from an existing Institutional Review Board-approved prospective, single-institution database. All women aged 45 years or younger with American Joint Committee on Cancer Stage II to III (Sixth Edition) breast cancer who underwent neoadjuvant chemotherapy from 1991 to 2007 were included. Patients presenting with Stage IV disease or with inflammatory breast cancer were excluded from the study.

All patients in the study underwent neoadjuvant chemotherapy after cancer diagnosis. The neoadjuvant regimens were selected at the discretion of the medical oncologist; however, most patients received an anthracycline, a taxane, or both. Hormone receptor-positive patients received adjuvant endocrine therapy, and radiation was administered at the discretion of the radiation oncologist.

Local-regional recurrence was defined as chest wall recurrence in patients undergoing mastectomy or ipsilateral breast recurrence in patients undergoing BCT and any regional lymph node basin (axillary, supraclavicular, or infraclavicular) recurrence. A recurrence in 
more than one of these locations simultaneously was counted as a single recurrence for the purposes of data collection and reporting.

\section{Statistical Methods}

The Kaplan-Meier method was used to generate and analyze survival curves for times to LRR and OS from the date of diagnosis. Follow-up extended past 10 years for some patients, but Kaplan-Meier plots are truncated at 5 years. Fisher exact and Wilcoxon tests were used to evaluate differences between surgery groups and differences in LRR rates.

\section{Results}

A total of 122 patients were included in the study (Table 1) and the median follow-up for survivors was 6.4 years. Fifty-four (44\%) patients had BCT and 68 (56\%) underwent mastectomy. Forty-nine per cent were white, 46 per cent black, and the median age was 39.5 years (range, 23 to 45 years). Most patients (89\%) had invasive ductal carcinoma, 3 per cent had invasive lobular carcinoma, and 8 per cent had cancers that were classified as other. Forty-six percent (52 of 113) were estrogen receptor-positive (ER+), 43 per cent (48 of 111) progesterone receptor-positive (PR+), and 31 per cent (28 of 90) overexpressed Her2. Immunophenotype was available on 90 patients with 39 per cent hormone receptor-positive and Her2-negative, 33 per cent ER/PR- and Her2-negative, 14 per cent hormone receptorpositive and Her2-positive, and 13 per cent ER/PR- and Her2-positive. Nineteen per cent of patients had lymphovascular invasion (LVI), 11 per cent in the BCT group and 25 per cent in the mastectomy group $(P=0.06)$. Mean pretreatment $\mathrm{T}$ size was $5.6 \mathrm{~cm}$ (range, 2.5 to 15 $\mathrm{cm})$ in the BCT group and $6.7 \mathrm{~cm}$ (range, 0 to $18 \mathrm{~cm}$ ) in the mastectomy group $(P=0.04)$ with 67 per cent of patients undergoing BCT having at least one positive node compared with 72 per cent of patients undergoing mastectomy $(P=0.6)$. The mean posttreatment $\mathrm{T}$ size was $1.3 \mathrm{~cm}$ (range, 0 to $8 \mathrm{~cm}$ ) in the BCT group and $3.2 \mathrm{~cm}$ (range, 0 to $10 \mathrm{~cm}$ ) in the mastectomy group $(P<0.0001)$.

\section{Treatment}

Ninety-seven per cent of patients underwent a regimen that included an anthracycline and 69 per cent of patients underwent a regimen that included a taxane. All but three patients who received a taxane also received an anthracycline. One patient did not receive either an anthracycline or a taxane.

Of the 25 patients who were Her2-positive, 40 per cent received trastuzumab. Fifty-seven $(47 \%)$ patients received endocrine therapy. The majority of patients received adjuvant radiation (87\%). Only one patients who underwent BCT and 15 patients who underwent mastectomy were not radiated (Table 2).

\section{Response}

The most prevalent clinical pretreatment stage was IIIA, which was the same in both the BCT and mastectomy groups (43 and 57\%, respectively). Pathologic posttreatment stages decreased with the highest proportion of patients undergoing BCT being Stage 0 (37\%) and only 13 per cent being Stage IIIA or higher post-treatment (Table 3). In the mastectomy 
group, only 32 per cent were Stage IIIA and 29 per cent were Stage 0 or I posttreatment. In the BCT group, there was a significant decrease in tumor size from a mean of 5.6 to $1.3 \mathrm{~cm}$ $(P<0.0001)$. There was also a significant decrease in the mastectomy group from 6.7 to 3.2 $\mathrm{cm}(P<0.0001)$.

Fifty-two patients (43\%) exhibited a clinical complete response (cCR) and 104 patients (87\%) exhibited either a clinical complete or partial response (cPR). Fifteen patients had stable disease and only one had progressive disease. There was no association between a cCR and time to local regional recurrence $(P=0.7)$, but those with a $\mathrm{cCR}$ did have better OS $(P=0.03)$. Eight patients $(15 \%)$ with a cCR had a LRR and taken together and 16 patients $(15 \%)$ with any response (cCR or cPR) had a LRR. Thirty-three patients (27\%) exhibited a pathologic complete response (21 (39\%) in the BCT group and 12 (18\%) patients undergoing mastectomy. Only three of these patients experienced a LRR (two in the BCT group and one in the mastectomy group).

\section{Local Regional Recurrence}

Of the 122 patients, 19 (16\%) had a local recurrence. LRR rates were no different after BCT compared with mastectomy (13 vs 18\%, $P=0.6$ ). In the BCT group, of the seven LRRs, six were isolated in breast tumor recurrences, and one had an isolated in breast tumor recurrences (IBTR) with a regional lymph node recurrence. In the mastectomy group, the 12 recurrences were as follows: eight isolated chest wall recurrences, one isolated regional lymph node, and three combined chest wall and regional lymph nodes. Additionally, LRR rates were no different between those with and without LVI, PR-positive and -negative, Her2-positive and -negative, molecular subtype, receipt of radiation, pathologic CR, or pretreatment stage (all $P>0.7$ ). Posttreatment stage was not associated with LRR rate $(P$ $=0.17)$, but posttreatment $\mathrm{N}$ stage was significantly associated $(P=0.02)$ with those who had pathologically positive nodes after chemotherapy more likely to develop a LRR. Fortytwo per cent ( 5 of 12) of those with pathologic N3 disease had a recurrence. For the 40 patients who had BRCA mutation analysis, 33 per cent ( 3 of 9$)$ of those with a mutation recurred compared with only 6 per cent ( 2 of 31 ) of those without the mutation $(P=0.07)$. Of the 9 patients with a mutation, six had a mastectomy (two with a LRR) and three had BCT (one LRR). Only 16 (13\%) of the patients were not radiated. Of the 19 LRRs, only three patients did not receive radiation to the site of recurrence. All three of these patients were mastectomy patients.

For the 19 patients who had a LRR, the median time to LRR was 1.7 years and ranged from 0.7 to 12.7 years. Overall, the 5-year recurrence-free rate was 85 per cent $(95 \%$ CI, 76.4 to $90.2 \%$ ) and 10-year recurrence-free rate was 82 per cent ( 72.6 to $88.9 \%$ ). There was no difference in time to local regional recurrence when comparing BCT with mastectomy (Fig. 1), but the patients undergoing BCT appear to do better with 5-year recurrence-free rates of 90 per cent compared with 81 per cent $(P=0.3)$. The only factors associated with time to LRR were posttreatment $\mathrm{N}$ stage $(P<0.001)$ and BRCA mutation status $(P=0.02)$. Eightyeight per cent of N0 to N2 patients were recurrence-free at 5 years compared to 50 per cent of N3 patients. Ninety-seven per cent of patients without BRCA mutations were recurrencefree at 5 years compared with 67 per cent of patients with BRCA mutations. 


\section{Overall and Disease-Free Survival}

Median follow-up of survivors was 6.4 years with 36 patients (30\%) dying. Of the 19 who had a LRR, $12(63 \%)$ were known to have died. Disease-free survival was better for patients achieving BCT versus patients undergoing mastectomy $(P=0.03)$ with 5-year DFS rates of 82 per cent (95\% CI, 69 to 90\%) compared with 58 per cent (95\% CI, 45 to 69\%). Median OS for the entire population was not reached, but the survival rate was 73 per cent at 5 years and 64 per cent at 10 years. Overall survival was significantly higher in patients achieving BCT compared with mastectomy $(P=0.004$, 5-year OS $88 v s 61 \%)$ (Fig. 2), without pathologic lymphovascular invasion $(P=0.001,5$-year OS 82 vs 49\%), having a CR compared with not $(P=0.03,5$-year OS 83 vs 68\%), and post $\mathrm{N}$ status 0 to 2 compared with $3(P<0.0001,5$-year OS 80 vs $18 \%)$.

\section{Discussion}

Neoadjuvant therapy has become a widely used and accepted treatment modality for locally advanced breast cancer, which can be used as a tool to allow women to undergo BCT when they may not have been optimal candidates at the time of cancer diagnosis. Multiple clinical trials, including the National Surgical Adjuvant Breast and Bowel B-18, have shown acceptable rates of recurrence and OS in patients treated with BCT after neoadjuvant chemotherapy. More patients were able to undergo BCT with 35 per cent of patients experiencing complete $\mathrm{CR}$ and 79 per cent of patients experiencing either complete $\mathrm{CR}$ or PR. ${ }^{6,10}$ Other studies have also shown similar rates of CR and PR and comparable recurrence rates among patients who undergo BCT after neoadjuvant therapy as compared with patients undergoing mastectomy. $7,8,11,12$

Despite the findings regarding BCT in the overall population after neoadjuvant therapy, there has been concern that young patients may not fare as well when undergoing BCT. Prior studies have demonstrated that young women with breast cancer are more likely to have a worse prognosis. Many studies have attributed this finding to a higher proportion of younger women being diagnosed with triple negative or Her2/neu-positive breast cancer when compared with breast cancer across the spectrum of ages. In the study by Hartley et al. in 2006, young women were found to have more ER/PR-negative tumors in addition to having an increased rate of Ki-67 staining and Her2 overexpression. ${ }^{13}$ As a result, young women are often counseled that they are at greater risk of experiencing higher disease recurrence after $\mathrm{BCT}$ despite response to neoadjuvant chemotherapy.

Studies of breast cancer recurrence with BCT followed by standard adjuvant therapy have shown that young women experience a higher rate of recurrence. The 20-year follow-up of the Milan trial demonstrated a rate of recurrence of 1.05 per 100 women-years in patients aged younger than 45 years after BCT compared with a recurrence rate of 0.34 per 100 women-years for women aged 45 to 60 years and a rate of 0.54 per 100 women-years for women age 60 years or older. When comparing BCT with mastectomy in the younger than 45 years age group, the rate of recurrence was higher in the patients undergoing BCT (1.05 per 100 women-years vs 0.18 per 100 women-years). ${ }^{2}$ Additionally, Kim et al. found that LRR rates of 16 per cent in young women 35 years or younger as compared with 11.5 per cent in older women. ${ }^{14}$ 
Our study demonstrates that young women may not experience an increased local recurrence rate after BCT if they have undergone neoadjuvant chemotherapy and were deemed to be candidates for BCT. Neoadjuvant therapy may select for chemotherapy-responsive tumor types with more favorable tumor biology, allowing women to undergo BCT without excessively increased risk of LRR. Additionally, DFS rates were improved for women who were able to undergo BCT after neoadjuvant chemotherapy as compared with women who underwent mastectomy, again suggesting that being a candidate for BCT after neoadjuvant chemotherapy may be a proxy for tumor biology.

We did not detect a difference in LRR in patients with a clinical or cPR, although theoretically it would be useful to correlate clinical response in young women to low risk of recurrence to be able to better stratify patients after neoadjuvant therapy because this may have an impact on clinical decision-making. Prior studies have shown a link between high pathologic CR and survival. ${ }^{7,15}$ This suggests, along with our findings, that young patients who are BCT candidates after neoadjuvant chemotherapy can proceed with BCT with local recurrence risk that are not excessive and are at least comparable to the risk associated with mastectomy.

\section{References}

1. Beadle BM, Woodward WA, Tucker SL, et al. Ten-year recurrence rates in young women with breast cancer by locoregional treatment approach. Int J Radiat Oncol Biol Phys. 2009; 73:734-44. [PubMed: 18707822]

2. Veronesi U, Cascinelli N, Mariani L, et al. Twenty-year follow-up of a randomized study comparing breast-conserving surgery with radical mastectomy for early breast cancer. N Engl J Med. 2002; 347:1227-32. [PubMed: 12393819]

3. Xiong Q, Valero V, Kau V, et al. Female patients with breast carcinoma age 30 years and younger have a poor prognosis: the M.D. Anderson Cancer Center experience. Cancer. 2001; 92:2523-8. [PubMed: 11745185]

4. Anders CK, Fan C, Parker JS, et al. Breast carcinomas arising at a young age: unique biology or a surrogate for aggressive intrinsic subtypes? J Clin Oncol. 2011; 29:e18-20. [PubMed: 21115855]

5. Anders CK, Hsu DS, Broadwater G, et al. Young age at diagnosis correlates with worse prognosis and defines a subset of breast cancers with shared patterns of gene expression. J Clin Oncol. 2008; 26:3324-30. [PubMed: 18612148]

6. Wolmark N, Wang J, Mamounas E, et al. Preoperative chemotherapy in patients with operable breast cancer: nine-year results from National Surgical Adjuvant Breast and Bowel Project B-18. J Natl Cancer Inst Monogr. 2001; 30:96-102. [PubMed: 11773300]

7. Makris A, Powles TJ, Ashley SE, et al. A reduction in the requirements for mastectomy in a randomized trial of neoadjuvant chemoendocrine therapy in primary breast cancer. Ann Oncol. 1998; 11:1179-84. [PubMed: 9862047]

8. Cance WG, Carey LA, Calvo BF, et al. Long-term outcome of neoadjuvant therapy for locally advanced breast carcinoma: effective clinical downstaging allows breast preservation and predicts outstanding local control and survival. Ann Surg. 2002; 236:295-302. [PubMed: 12192316]

9. Chen AM, Meric-Bernstam F, Hunt KK, et al. Breast conservation after neoadjuvant chemotherapy: the M.D Anderson Cancer Center experience. J Clin Oncol. 2004; 22:2303-12. [PubMed: 15197191]

10. Fisher ER, Wang J, Bryant J, et al. Pathobiology of preoperative chemotherapy: findings from the National Surgical Adjuvant Breast and Bowel (NSABP) protocol B-18. Cancer. 2002; 95:681-95. [PubMed: 12209710] 
11. Fisher B, Brown A, Mamounas E, et al. Effect of preoperative chemotherapy on local-regional disease in women with operable breast cancer: findings from National Surgical Adjuvant Breast and Bowel Project B-18. J Clin Oncol. 1997; 15:2483-93. [PubMed: 9215816]

12. Huang EH, Strom EA, Perkins GH, et al. Comparison of risk of local-regional recurrence after mastectomy or breast conservation therapy for patients treated with neoadjuvant chemotherapy and radiation stratified according to a prognostic index score. Int J Radiat Oncol Biol Phys. 2006; 66:352-7. [PubMed: 16887286]

13. Hartley MC, McKinley BP, Rogers EA, et al. Differential expression of prognostic factors and effect on survival in young $(<$ or $=40)$ breast cancer patients: a case-control study. Am Surg. 2006; 72:1189-94. [PubMed: 17216817]

14. Kim SH, Simkovich-Heerdt A, Tran KN, et al. Women 35 years of age or younger have higher locoregional relapse rates after undergoing breast conservation therapy. J Am Coll Surg. 1998; 187:1-8. [PubMed: 9660018]

15. Rastogi P, Anderson SJ, Bear HD, et al. Preoperative chemotherapy: updates of National Surgical Adjuvant Breast and Bowel Project Protocols B-18 and B-27. J Clin Oncol. 2008; 26:778-85. [PubMed: 18258986] 


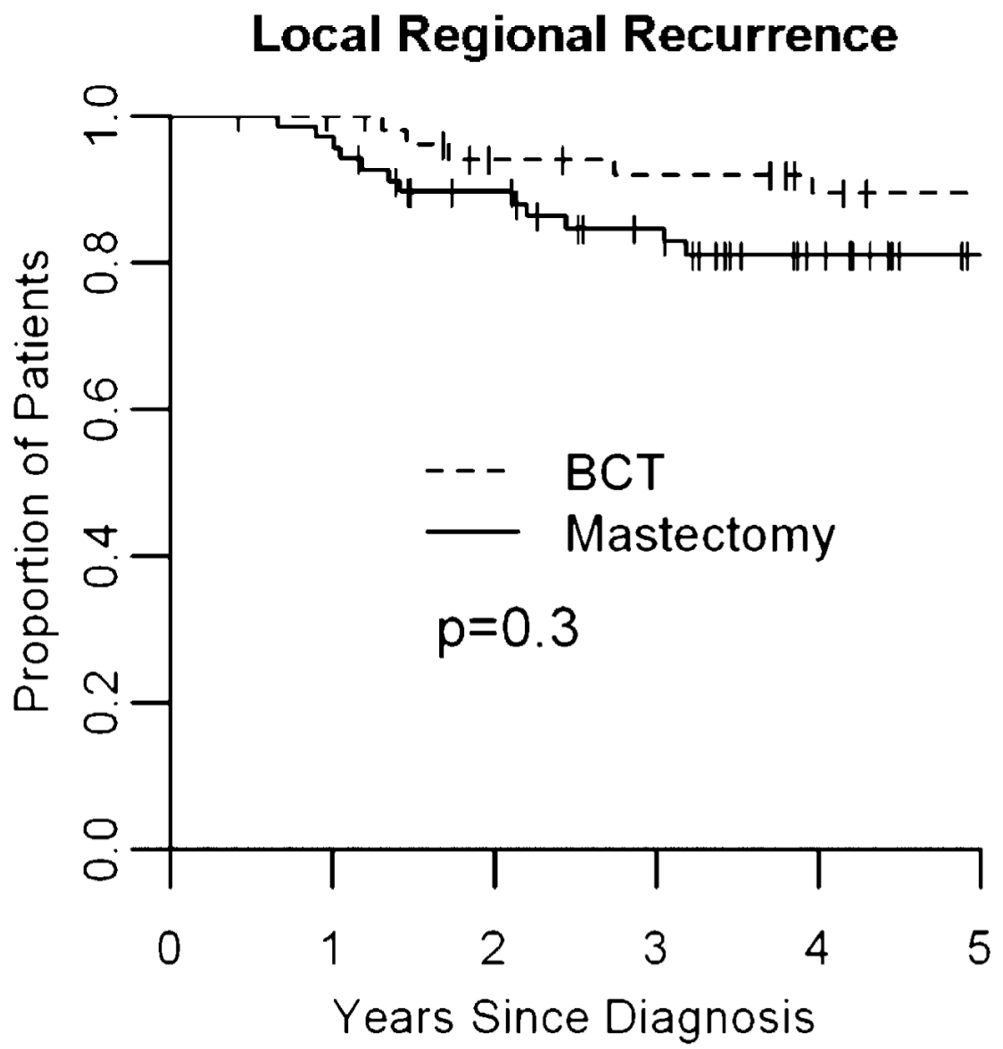

Fig. 1.

Time to local regional recurrence in patients undergoing breast conservation (BCT) versus mastectomy. The Kaplan-Meier method was used to generate and analyze survival curves for times to local regional recurrence from date of diagnosis. Time to local regional recurrence was no different after BCT versus mastectomy (5-year local regional recurrencefree rates 90 vs $81 \%, P=0.3$ ). 


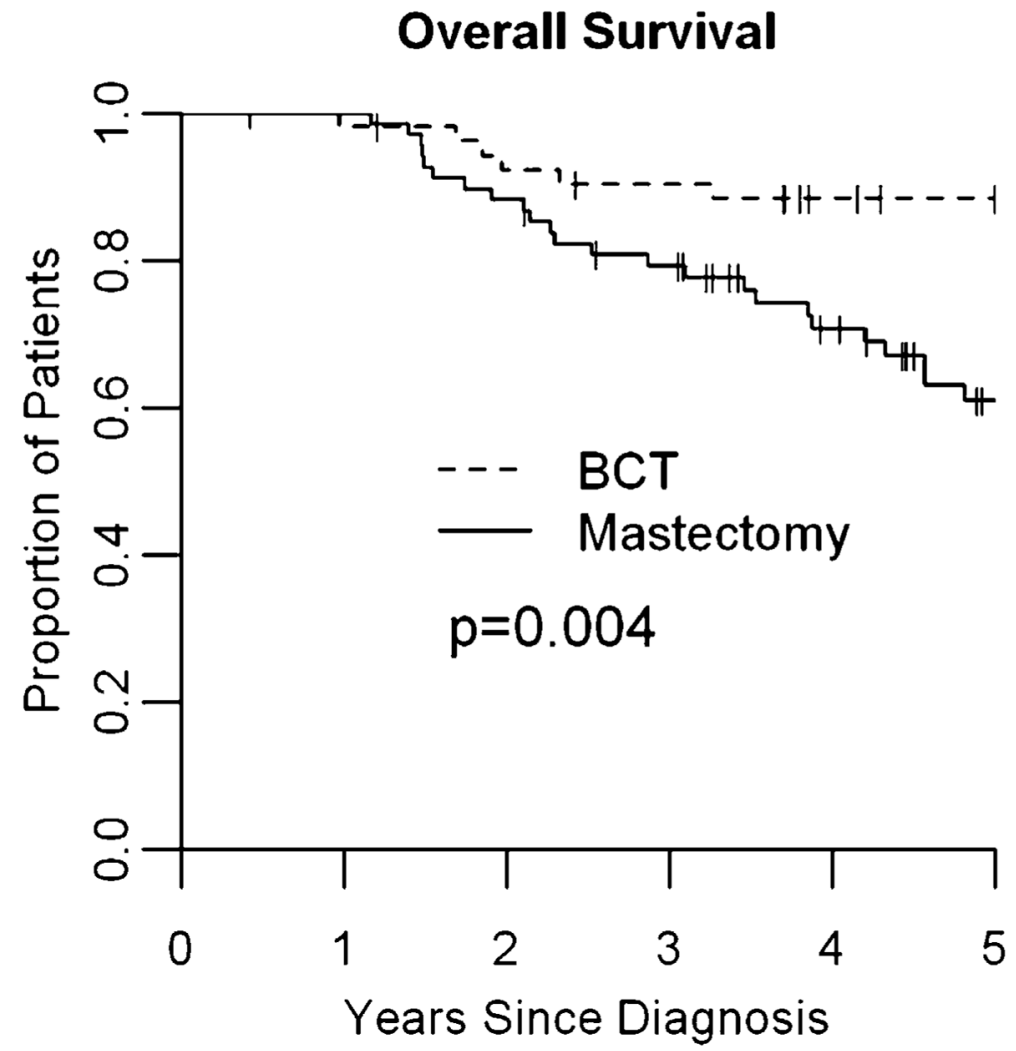

Fig. 2.

Time to overall survival in patients undergoing breast conservation (BCT) versus mastectomy. The Kaplan-Meier method was used to generate and analyze survival curves for times to overall survival from date of diagnosis. Overall survival was significantly higher in patients achieving BCT compared with mastectomy $(P=0.004,5$-year overall survival 88 vs $61 \%)$. 


\section{Table 1}

Demographics and Pretreatment Clinical Stage

\begin{tabular}{|c|c|c|c|c|}
\hline & Total & BCT & Mastectomy & $P$ \\
\hline Patients & 122 & $54(44 \%)$ & $68(56 \%)$ & \\
\hline Median age (years) (range) & $39(23-45)$ & $39(23-45)$ & $40(23-45)$ & 0.8 \\
\hline \multicolumn{5}{|l|}{ Race } \\
\hline White & $60(49 \%)$ & $27(50 \%)$ & $33(48 \%)$ & 0.9 \\
\hline Black & $56(46 \%)$ & $25(46 \%)$ & $31(46 \%)$ & \\
\hline Other & $6(5 \%)$ & $2(4 \%)$ & $4(6 \%)$ & \\
\hline \multicolumn{5}{|c|}{ American Joint Committee on Cancer stage } \\
\hline I & $2(2 \%)$ & $0(0 \%)$ & $2(3 \%)$ & 0.4 \\
\hline IIA & $18(15 \%)$ & $11(20 \%)$ & $7(10 \%)$ & \\
\hline IIB & $25(20 \%)$ & $12(22 \%)$ & $13(19 \%)$ & \\
\hline IIIA & $62(51 \%)$ & $23(43 \%)$ & $39(58 \%)$ & \\
\hline IIIB & $11(9 \%)$ & $6(11 \%)$ & $5(7 \%)$ & \\
\hline IIIC & $4(3 \%)$ & $2(4 \%)$ & $2(3 \%)$ & \\
\hline \multicolumn{5}{|l|}{$\mathrm{T}$ stage } \\
\hline $\mathrm{T} 1$ & $4(3 \%)$ & $0(0 \%)$ & $4(6 \%)$ & 0.03 \\
\hline $\mathrm{T} 2$ & $39(32 \%)$ & $23(43 \%)$ & $16(24 \%)$ & \\
\hline $\mathrm{T} 3$ & $67(56 \%)$ & $25(46 \%)$ & $42(63 \%)$ & \\
\hline $\mathrm{T} 4$ & $11(9 \%)$ & $6(11 \%)$ & $5(7 \%)$ & \\
\hline \multicolumn{5}{|l|}{$\mathrm{N}$ stage } \\
\hline No & $37(30 \%)$ & $18(33 \%)$ & $19(28 \%)$ & 0.8 \\
\hline N1 & $61(50 \%)$ & $27(50 \%)$ & $34(50 \%)$ & \\
\hline $\mathrm{N} 2$ & $20(76 \%)$ & $7(13 \%)$ & $13(19 \%)$ & \\
\hline N3 & $4(3 \%)$ & $2(4 \%)$ & $2(3 \%)$ & \\
\hline \multicolumn{5}{|l|}{ Histology } \\
\hline IDC & $109(89 \%)$ & $45(83 \%)$ & $64(94 \%)$ & 0.14 \\
\hline ILC & $3(3 \%)$ & $2(4 \%)$ & $1(2 \%)$ & \\
\hline Other & $10(8 \%)$ & $7(13 \%)$ & $3(4 \%)$ & \\
\hline \multicolumn{5}{|l|}{ ER status } \\
\hline Positive & $52(46 \%)$ & $20(42 \%)$ & $32(49 \%)$ & 0.5 \\
\hline Negative & $61(54 \%)$ & $28(58 \%)$ & $33(51 \%)$ & \\
\hline \multicolumn{5}{|l|}{ PR status } \\
\hline Positive & $48(43 \%)$ & $19(40 \%)$ & $29(45 \%)$ & 0.7 \\
\hline Negative & $63(57 \%)$ & $28(60 \%)$ & $35(55 \%)$ & \\
\hline \multicolumn{5}{|l|}{ Her2 status } \\
\hline Overexpressed/borderline & $28(31 \%)$ & $9(27 \%)$ & $19(33 \%)$ & 0.6 \\
\hline Negative & $62(69 \%)$ & $24(73 \%)$ & $38(67 \%)$ & \\
\hline
\end{tabular}

BCT, breast conservation; IDC, invasive ductal carcinoma; ILC, infiltrating lobular carcinoma; ER, estrogen receptor; PR, progesterone receptor. 
Table 2

Proportion of Patients Receiving Systemic Therapy and Radiation Therapy

\begin{tabular}{lrrrc}
\hline Chemotherapy & BCT $(\mathbf{n}=\mathbf{5 4})$ & Mastectomy $(\mathbf{n}=\mathbf{6 8})$ & \multicolumn{1}{c}{ Total } & \multicolumn{1}{c}{$\boldsymbol{P}$} \\
\hline Anthracycline & $52(96 \%)$ & $66(97 \%)$ & $118(97 \%)$ & 0.99 \\
Taxane* $^{*}$ & $33(61 \%)$ & $51(75 \%)$ & $84(69 \%)$ & 0.11 \\
Biologic therapy received (Trastuzumab) & $4(44 \%)$ & $6(38 \%)$ & $10(8 \%)$ & 0.99 \\
Endocrine therapy received & $18(33 \%)$ & $39(57 \%)$ & $57(47 \%)$ & 0.01 \\
Radiation received & $53(98 \%)$ & $53(78 \%)$ & $106(87 \%)$ & $<0.001$ \\
\hline
\end{tabular}

* All patients receiving a taxane also received an anthracycline.

$\mathrm{BCT}$, breast conservation. 
Table 3

Comparison of Pretreatment Clinical Stage with Pathologic Response to Chemotherapy

\begin{tabular}{|c|c|c|c|c|}
\hline & \multicolumn{2}{|c|}{ Pretreatment Clinical Stage } & \multicolumn{2}{|c|}{ Posttreatment Pathologic Stage } \\
\hline & BCT & Mastectomy & BCT & Mastectomy \\
\hline \multicolumn{5}{|l|}{ Stage } \\
\hline 0 & $0(0 \%)$ & $0(0 \%)$ & $20(37 \%)$ & $9(13 \%)$ \\
\hline I & $0(0 \%)$ & $2(3 \%)$ & $14(26 \%)$ & $11(16 \%)$ \\
\hline IIA & $11(20 \%)$ & $7(10 \%)$ & $10(19 \%)$ & $10(15 \%)$ \\
\hline IIB & $12(22 \%)$ & $13(19 \%)$ & $3(6 \%)$ & $5(7 \%)$ \\
\hline IIIA & $23(43 \%)$ & $39(57 \%)$ & $2(4 \%)$ & $22(32 \%)$ \\
\hline IIIB & $6(11 \%)$ & $5(7 \%)$ & $2(4 \%)$ & $2(3 \%)$ \\
\hline IIIC & $2(4 \%)$ & $2(3 \%)$ & $3(6 \%)$ & $9(13 \%)$ \\
\hline \multicolumn{5}{|l|}{ T stage } \\
\hline 0 & $0(0 \%)$ & $0(0 \%)$ & $16(30 \%)$ & $8(12 \%)$ \\
\hline 1 & $0(0 \%)$ & $4(6 \%)$ & $24(44 \%)$ & $22(32 \%)$ \\
\hline 2 & $23(43 \%)$ & $16(24 \%)$ & $5(9 \%)$ & $12(18 \%)$ \\
\hline 3 & $25(46 \%)$ & $42(62 \%)$ & $2(4 \%)$ & $19(28 \%)$ \\
\hline 4 & $6(11 \%)$ & $5(7 \%)$ & $2(4 \%)$ & $3(4 \%)$ \\
\hline Tis & $0(0 \%)$ & $1(1 \%)$ & $5(9 \%)$ & $4(6 \%)$ \\
\hline \multicolumn{5}{|l|}{$\mathrm{N}$ stage } \\
\hline 0 & $18(33 \%)$ & $19(28 \%)$ & $37(69 \%)$ & $29(43 \%)$ \\
\hline 1 & $27(50 \%)$ & $34(50 \%)$ & $10(19 \%)$ & $14(21 \%)$ \\
\hline 2 & $7(13 \%)$ & $12(19 \%)$ & $4(7 \%)$ & $16(24 \%)$ \\
\hline 3 & $2(4 \%)$ & $2(3 \%)$ & $3(6 \%)$ & $9(13 \%)$ \\
\hline Tumor size $(\mathrm{cm})$ (mean) & 5.6 & 6.7 & 1.3 & 3.2 \\
\hline
\end{tabular}

$\mathrm{BCT}$, breast conservation. 